\title{
Demografía de la insuficiencia cardiaca en Galicia. Análisis de diferentes poblaciones y ámbitos asistenciales
}

\author{
Heart failure demographics in Galicia. \\ An analysis of diverse populations and care settings
}

\author{
Julio Montes-Santiago ${ }^{1}$, Marta Lado Castro-Rial' ${ }^{1}$ Gloria Rey García², Ana Mediero Domínguez² , Roberto Pérez Álvarez \\ Servicios de Medicina Interna', Documentación Clínica². Complejo Hospitalario Universitario-Meixoeiro. Vigo.
}

\begin{abstract}
Resumen
Objetivo. Galicia es la Autonomía española con mayor tasa de mortalidad por insuficiencia cardiaca (IC). En los últimos años se han realizado en Galicia investigaciones en diferentes ámbitos asistenciales para intentar perfilar las características de dicho proceso. El objetivo de este análisis fue revisar y comparar las características demográficas de la IC en dichos estudios.

Material y métodos. Se estudiaron las características demográficas comunes al menos a tres de los siguientes estudios recientes: INCARGAL (951 pacientes ingresados en Medicina Interna-Geriatría o Cardiología); Hospital Clínico Universitario de Santiago (Card-CHUS)(1252 pacientes en Cardiología, periodo 1991-2002); GALICAP (1195 pacientes ingresados, seguidos posteriormente en Atención Primaria), GALICEP (25487 altas de Hospitales del SERGAS, periodo 2002-2005).
\end{abstract}

Resultados. Edad media (años): INCARGAL: 75. (M. InternaGeriatría: 77, Cardiología: 72), Card-CHUS: 64, GALICAP: 76. Porcentaje>75 años: Card-CHUS: 33\%, GALICAP (>70): 79\%, GALICEP: $65 \%$. Mujeres: INCARGAL: 52\% (M. Interna-Geriatría: 56\%, Cardiología: 48\%), Card-CHUS: 37\%, GALICAP: 52\% GALICEP: 59\%); HTA (antecedentes): INCARGAL: 59\%, Card-CHUS: 55\%, GALICAP: 82\%. HTA (etiología): GALICAP: 36\%, GALICEP: 20\%. Días Estancia: INCARGAL: 11, Card-CHUS: 14, GALICEP: 10. Mortalidad hospitalaria: INCARGAL: 7\%, Card-CHUS (12 meses): 16\%, GALICEP: $10 \%$.

Conclusiones. Cerca de 2 de cada 3 pacientes con IC en Galicia son $>75$ años. Los pacientes ingresados en Cardiología son de menor edad y en mayor proporción varones. La mayoría de IC presentan HTA como antecedente o causa etiológica. Los días de estancia oscilaron entre 10-14 días y se observó una moderada mortalidad hospitalaria (entre el $7-10 \%)$.

\section{Abstract}

Background. Galicia has the greatest mortality due to heart failure (HF) in Spain. In the latest years some studies have tried to outline the characteristics of these process. Our purpose was to analyze and compare the recent published demographic data of HF in Galicia.

Material and methods. Common demographic characteristics to three out the four recent available studies were considered. INCARGAL (951 inpatients at Internal Medicine (IM)-Geriatrics or Cardiology wards); Study of the Cardiology Service of the University Clinical Hospital of Santiago (Card-CHUS)(1252 inpatients followed in the period 19912002); GALICAP (1195 inpatients with HF, evaluated later in the primary care setting), GALICEP (25487 discharges of HF patients in Galician hospitals in the period 20022005).

Results. Mean (years): INCARGAL (Global): 75(IM-Geriatrics: 77. Cardiology: 72), Card-CHUS: 64, GALICAP: 76 . Age $>75$ years (\%): Card-CHUS: 33\%, GALICAP (>70): 79\%, GALICEP: 65\%. Women: INCARGAL (Global): 52\%(IM-Geriatrics: 56\%, Cardiology: 48\%), Card-CHUS: 37\%, GALICAP: 52\% GALICEP: 59\%). Hypertension (antecedent): INCARGAL: 59\%, Card-CHUS: 55\%, GALICAP: 82\%. HTA (etiology): GALICAP: $36 \%$, GALICEP: 20\%. Stay (days): INCARGAL: 11. Card-CHUS: 14, GALICEP: 10. Inhospital mortality: INCARGAL: 7\%, Card-CHUS (at 12 months): 16\%, GALICEP: $10 \%$.

Conclusions. Nearly 2 out of 3 inpatients with HF in Galicia are older than 75 years. Cardiology-attended inpatients are younger and in greater proportion men. Most patients are hypertensive. The duration of hospitalization ranged 10-14 days and a moderate inhospital mortality rate was observed (7-10\%).

Key words: Heart failure. Galicia.

Palabras clave: Insuficiencia cardiaca. Galicia. 


\section{Introducción}

Galicia es la Comunidad Autónoma española con mayor tasa de mortalidad por insuficiencia cardiaca (IC)[Año 2004: Tasa bruta por 100000 h: 64,6 (Galicia) vs. 43,4 (España)]' ${ }^{1}$ Aunque este dato es parcialmente atribuible al mayor envejecimiento de la población gallega, no explica totalmente la situación, puesto que dichas tasas superiores de mortalidad de Galicia siguen manteniéndose al realizar el ajuste por edad (Galicia: 44,9 vs. 40,1 resto de España) ${ }^{1}$. Ello hace que esta entidad, que constituye la $3^{\mathrm{a}}$ causa de mortalidad en esta comunidad, constituya un importante problema, tanto asistencial como económico². Estimaciones recientes, por ejemplo, han situado los gastos totales asistenciales de la IC en Galicia -intra y extrahospitalarios, además de los domiciliarios para aquellos pacientes hospitalizados en 2004- en 173 millones $€(0,64 \% \text { del presupuesto global del SERGAS })^{3}$.

En los últimos años se han realizado diversos estudios en pacientes con IC hospitalizados en Galicia con el fin de perfilar sus características, con frecuencia procedentes de un único centro ${ }^{3-7}$. Se ha demostrado que en esta Comunidad existen ciertas disparidades geográficas provinciales e incluso locales en cuanto a la hospitalización de pacientes con IC ${ }^{8}$. Por ello, y para intentar evitar dichos sesgos locales, en este análisis sólo se revisarán aquellos estudios multicéntricos gallegos o bien aquellos que, aunque procedentes de un único centro, hubieran reclutado a más de 1000 pacientes durante un periodo prolongado de tiempo. El objetivo de este análisis fue, pues, comparar las características demográficas de grupos representativos de pacientes con IC estudiados recientemente en diversos ámbitos asistenciales de Galicia.

\section{Material y métodos}

Se estudiaron y compararon aquellas características demográficas que aparecieran reflejadas en al menos tres de los cuatro estudios, recientemente disponibles y cuyas caracte- rísticas se describen a continuación (por orden cronológico de comunicación-publicación de resultados):

Estudio INCARGAL (Insuficiencia cardiaca en Galicia): Estudio descriptivo trasversal multicéntrico durante 1999 de 951 pacientes ingresados en Servicios de Medicina Interna y/o Geriatría (587) o Cardiología (364) de 14 hospitales públicos.

Estudio del Servicio de Cardiología del Hospital Clínico Universitario de Santiago $(\mathrm{Card}-\mathrm{CHUS})^{10}$. Estudio prospectivo longitudinal de 1252 pacientes ingresados en dicho servicio durante un periodo de 12 años (1991-2002).

Estudio GALICAP (Estudio gallego de insuficiencia cardiaca en atención primaria) ${ }^{11}$ : Estudio descriptivo transversal multicéntrico de 1195 pacientes ingresados por IC en 2004 y seguidos posteriormente por 149 médicos en Atención Primaria (media de 8 pacientes por médico).

Proyecto GALICEP (Epidemiología de insuficiencia cardiaca en Galicia) ${ }^{12}$ : Estudio descriptivo transversal multicéntrico de 25487 altas de pacientes con IC residentes en Galicia (media de 6372 altas anuales) procedentes del Conjunto Mínimo Básico de Datos al Alta hospitalaria (CMBDAH) de todos los hospitales del SERGAS. Se estudiaron los Grupos Relacionados por el Diagnóstico (GRD) específicos de IC (127 y 544) durante el periodo 2002-2005.

\section{Resultados}

En la tabla 1 se detallan ciertas características diferenciales demográficas de la población general y de la hospitalizada por IC en Galicia con respecto a la española. Ha de observarse el mayor envejecimiento de la población gallega lo cual se traduce en el mayor porcentaje de altas en pacientes $>65$ años. Correspondiendo a una patología de presentación preferente en pacientes de edad avanzada el porcentaje de altas con el diagnóstico de IC es mayor en Galicia que en el

\section{Tabla 1}

Comparación entre las características demográficas generales y de hospitalización por Insuficiencia Cardiaca (IC) entre Galicia y España (2004). (Elaboración propia a partir de ref. ${ }^{1 \text { y }{ }^{13} \text { ) }}$

\begin{tabular}{|c|c|c|}
\hline Datos demográficos (2004) & Galicia & España \\
\hline Población > 65 años & $21,3^{\star}$ & 16,6 \\
\hline$\%$ altas hospitalarias >65 años & $41,5^{\star}$ & 36,9 \\
\hline \multicolumn{3}{|l|}{ Datos epidemiológicos de IC } \\
\hline Tasa de altas IC por $100000 \mathrm{~h}$. & $249^{*}$ & 209 \\
\hline$\%$ altas por $I C>65$ años & 88,6 & 88,5 \\
\hline$\%$ de IC en altas >65 años & $6,1^{*}$ & 4,4 \\
\hline Tasa de altas IC por $100000 \mathrm{~h}>65$ años & 1051 & $1091^{*}$ \\
\hline$\%$ muertes por IC >65 años & 96,5 & 95,4 \\
\hline Tasa bruta de mortalidad por IC por $100000 \mathrm{~h}$. & $66^{*}$ & 45 \\
\hline Tasa ajustada por edad de mortalidad por IC por 100000 h. & 45 & 41 \\
\hline Tasa global de mortalidad por $100000 \mathrm{~h}>65$ años & $304^{*}$ & 262 \\
\hline \multicolumn{3}{|l|}{${ }^{*} \mathrm{p}<0,01$} \\
\hline
\end{tabular}


Tabla 2

Características demográficas de los estudios sobre poblaciones gallegas con insuficiencia cardiaca ${ }^{9-12}$

\begin{tabular}{|c|c|c|c|c|c|c|}
\hline & \multicolumn{3}{|c|}{ INCARGAL } & \multirow{2}{*}{ CARD-CHUS } & \multirow{2}{*}{ GALICAP } & \multirow{2}{*}{ GALICEP } \\
\hline & MI-Geriatría & Cardiología & Global & & & \\
\hline $\mathrm{N}^{0}$ & 587 & 364 & 951 & 1252 & 1195 & 25487 \\
\hline Edad media & 77 & 73 & 76 & 64 & 76 & -- \\
\hline >75 años (\%) & & & 75 & 33 & $79^{*}$ & 66 \\
\hline Mujeres (\%) & 56 & 48 & 52 & 37 & 52 & 59 \\
\hline HTA (antecedente) & 62 & 54 & 59 & 55 & 82 & -- \\
\hline HTA (etiología) & -- & -- & -- & & 36 & 20 \\
\hline Días estancia & 11 & 11 & 11 & 14 & -- & 10 \\
\hline Mortalidad & 7,0 & 6,6 & 6,8 & $16^{\star \star}$ & & 10,2 \\
\hline
\end{tabular}

resto de España (6,1\% vs. 4,4\%)13. En ambas el $89 \%$ de la población ingresada con IC es >65 años.

Dado que en este análisis se estudiaron los rasgos demográficos comunes a los estudios, se excluyeron los datos de interés aportados por los estudios individuales (p. ej. comorbilidad, situación familiar, etc.) pero no disponibles en todos ellos. En la tabla 2 se reflejan las características comunes de los estudios analizados. Aunque la diversidad de las poblaciones estudiadas, la forma heterogénea de comunicar los resultados y las fuentes utilizadas no aconsejan comparaciones estadísticas directas, pueden extraerse de este análisis algunas conclusiones razonables. Los pacientes ingresados en los Servicios de Cardiología son de menor edad y en mayor proporción varones. La HTA se recoge como antecedente en la mayoría de los pacientes, aunque la adscripción etiológica de la IC a la HTA se consigna en menor proporción de los casos. La mortalidad durante un ingreso por IC es variable pero indica que 1 de cada 10 ó 12 pacientes fallece durante el mismo.

\section{Discusión}

En este estudio se han analizado las características demográficas de los pacientes ingresados con IC en Galicia en los últimos años. La procedencia de los datos de diversas fuentes que pueden considerarse como complementarias, la publicación en fechas muy reciente de estos estudios y el gran número de pacientes analizados, nos permite afirmar que en el momento actual Galicia se encuentra en una posición privilegiada en cuanto al conocimiento de las características de los pacientes hospitalizados por IC en dicha comunidad. Una objeción que puede hacerse a este estudio es que, dado que la IC es un diagnóstico clínico, algunos de los pacientes ingresados podrían haber sido diagnosticados incorrectamente de IC. En este sentido debe recalcarse, por ejemplo, que la realización del ecocardiagrama es baja, sobre todo en Ios Servicios de Medicina Interna. Por ejemplo, en el estudio
INCARGAL ${ }^{9}$ sólo el 54\% de los pacientes (505/946) tenían ecocardiograma en el ingreso actual 0 en previos (41\% en MI-Geriatría, $74 \%$ en Cardiología) $)^{9}$. No obstante, debe hacerse notar que dicho estudio se realizó antes de que en las actuales Guías de Diagnóstico y Tratamiento de la Insuficiencia cardiaca crónica, ahora de amplia difusión ${ }^{14-16}$, se exigiera como condición inexcusable para sustanciar tal diagnóstico la valoración de la función ventricular izquierda (FEVI), usualmente mediante ecocardiograma. En el estudio GALICAP, realizado después de esta fecha, tenían realizado ecocardiograma el $68 \%$ de los pacientes ${ }^{11}$. Por otra parte, estos porcentajes son similares a la de otros registros realizados en periodos semejantes de tiempo, tanto nacionales (Estudio SEMI-IC: 2145 pacientes, ecocardiograma: 72\%) ${ }^{17}$ como europeos (EUROHEART FAILURE SURVEY: 11327 pacientes, ecocardiograma: $66 \%)^{18}$. Aun reconociendo tal limitación, que debe mejorarse pues se ha demostrado que el ajuste a las recomendaciones establecidas en las Guías mejora el pronóstico ${ }^{19}$, ello indica que el presente estudio en Galicia no parece presentar importantes sesgos de selección en cuanto al diagnóstico.

Con respecto a los resultados cabe destacar que los pacientes ingresados con IC en Galicia son de avanzada edad pues 2 de cada 3 pacientes son $>75$ años y predominan las mujeres. Ello es especialmente evidente en los Servicios de Medicina Interna en que la mayor parte de ellos son ya octogenarios, y por tanto con acusados componentes de otros procesos comórbidos o potenciales toxicidades medicamentosas. Este estudio viene a confirmar en los aspectos demográficos, las diferencias en las poblaciones atendidas por los diversos especialistas y que deberán tenerse en cuenta en las planificaciones asistenciales futuras para esta entidad. Como se ha comprobado en anteriores ocasiones, los pacientes que ingresan en los servicios de Cardiología son más jóvenes, con mayor frecuencia varones, con la cardiopatía isquémica como etiología predominante y mayor proporción 
de pacientes con FEVI deprimida. Aunque algunos análisis indican que las diferencias por sexo y edad pueden estar enjugándose entre especialidades ${ }^{20,21}$, en el momento actual los estudios publicados, al menos en España, indican que estas discrepancias en la presentación hospitalaria se mantienen también en la asistencia ambulatoria. Así en el estudio BADAPIC que recogió los datos de 3909 pacientes atendidos en consultas ambulatorias de Cardiología especialmente focalizadas en IC, la edad media fue de 66 años, sólo un tercio fueron mujeres y el $40 \%$ presentaba una etiología isquémi$\mathrm{ca}^{22}$. En Asistencia Primaria (AP), por el contrario, y de forma parecida a lo que ocurre en Medicina Interna ${ }^{23}$, los pacientes son de edad más avanzada, en mayor proporción mujeres y fundamentalmente hipertensos. En este ámbito asistencial al estudio gallego GALICAP (1195 pacientes, edad: 76 años, $52 \%$ mujeres) y al nacional CARDIOTENS (asistencia ambulatoria cardiológica y de AP, 1420 pacientes, edad: 71 años, $53 \%$ mujeres $)^{24}$, hay que añadir los datos similares recientes del estudio nacional CARDIOPRES (asistencia en AP, 847 pacientes, edad: 73 años, 49,5\% mujeres) ${ }^{25}$.

Se evidencia que la mayor parte de los pacientes que ingresan por IC en Galicia presentan antecedentes de HTA, aunque la atribución etiológica de la IC a HTA se refleja en un número mucho menor de casos. La estancia media hospitalaria en Galicia (10-14 días) es superponible a la otros registros multicéntricos hospitalarios (SEMI-IC: 10,2 días) ${ }^{17}$ y del SNS [Encuesta de morbilidad hospitalaria (EMH-2005): $9,5$ días $]^{13}$. En cuanto a la mortalidad intrahospitalaria osciló en Galicia ente el 6,8-10,2\%, también en los rangos comunicados por Registros españoles (SEMI-IC: 6,1\%) ${ }^{18} 0$ del SNS (EMH-2005: 88926 altas, mortalidad: 10,8\%) ${ }^{13}$.

Como conclusiones, pues, se puede decir que aproximadamente 2 de cada 3 pacientes con IC en Galicia son $>75$ años, aunque los pacientes ingresados en Cardiología tienden a ser más jóvenes y en mayor proporción varones. En una mayoría de pacientes la HTA se recoge como antecedente y/o causa etiológica. Los días de estancia oscilaron entre 10-14 días y se observó una moderada mortalidad hospitalaria (entre el 7-10\%).

\section{Bibliografía}

1. Xunta de Galicia. Plan de Saúde de Galicia 2006/2010. Consellería de Sanidade. Dirección General de Aseguramento e Planificación sanitaria, CD-ROM, 2006.

2. Montes Santiago J, Rey García G, Mediero Domínguez A. Costes directos de las hospitalizaciones por insuficiencia cardiaca crónica de origen hipertensivo en Galicia. Revista Galega Actualidade Sanitaria 2001;1(3):170-73.

3. Montes-Santiago J, Rey García G, Mediero Domínguez A. Economic burden of heart failure in Galicia (Northwest of Spain): The GALICEP-costes study. European Federation of Internal Medicine, 6th Congress. Lisbon 2007(May 23-26); Abstract book: P-49-07.

4. Ramos Polledo V, Pita Fernández S, de la Iglesia Martínez F, Pellicer Vázquez C, Nicolás Miguel R, Diz-Lois Martínez F, et al. Etiología, características clínicas, causa desencadenante, tipo de disfunción ventricular, estancia media y mortalidad de 305 pacientes ingresados por insuficiencia cardíaca. An Med Interna (Madrid) 2000;17:19-24.

5. Montes Santiago J, Rey García G, Mediero Domínguez A, González Vázquez L, Pérez Fernández E, del Campo Pérez V, Pérez Álvarez R. Variaciones estacionales en la hospitalización y mortalidad por insuficiencia cardiaca crónica en Vigo. An Med Interna (Madrid) 2001;18:578-81.
6. De Toro Santos M, López Castro J, Pérez de Juan Romero M, Gayoso Diz P, González Juanatey JR: Estudio epidemiológico de la insuficiencicia cariaca según género y grupo etario en la provincia de Ourense (Cohorte de la comunidad gallega). Arch Medicina (online) 2005; 1(4): 1-13. Disponible en: http://archivosdemedicina.com, [consultado, 12-01-2008].

7. Cinza Sanjurjo S, Cabarcos Ortiz de Barrón A, Nieto Pol E, Torre Carballada JA. Análisis transversal de la insuficiencia cardiaca en pacientes de un servicio de medicina interna de un hospital de tercer nivel de área mixta (rural y urbana). Parte I: análisis epidemiológico. An Med Interna (Madrid). 2007;24:267-72.

8. Montes-Santiago J, Rey-García G, Mediero-Domínguez A. Diferencias geográficas locales en las altas por insuficiencia cardiaca en ancianos en un área de Galicia. Comparación con la hospitalización convencional. Rev Clin Esp. 2006;206 (Supl. 3):152.

9. García Castelo A, Muñiz García J, Sesma Sánchez P, Castro Beiras A. Utilización de recursos diagnósticos y terapéuticos en pacientes ingresados por insuficiencia cardiaca: influencia del servicio de de ingreso (estudio INCARGAL). Rev Esp Cardiol. 2003;56:49-56.

10. Varela-Román A, Grigorian Shamagian L, Barge E, Bassante P, De la Peña MG, González-Juanatey JR. Heart failure in patients with preserved and deteriorated left ventricular ejection fraction: long term prognosis. Heart. 2005;91:489-94.

11. Otero-Raviña F, Grigorian-Shamagian L, Fransi-Galiana L, Názara-Otero C, Fernández-Villaverde JM, Del Álamo Alonso A et al. Estudio gallego de insuficiencia cardiaca en atención primaria (estudio GALICAP). Rev Esp Cardiol. 2007;60:373-83.

12. Montes-Santiago J, Rey-García G, Mediero-Domínguez A. El proyecto GALICEP: Epidemiología de la insuficiencia cardiaca en el anciano en Galicia. Variaciones geográficas provinciales. Rev Clin Esp 2006;206 (Supl. 3):160.

13. Instituto Nacional de Estadística. Cifras oficiales de población. Disponible en: http://www.ine.es/inebase/cgi/axi. Principales causas de muerte por comunidades autónomas de residencia (2004). Encuesta de Morbilidad Hospitalaria, 2004 y 2005. Disponible en: http://www.ine.es/jaxi/tabla.do?path=/t15/p414/a2005/ I0/\&file=01001.px\&type=pcaxis. [Consultados, 15-01-2008].

14. Remme WJ, Swedberg K.. Task Force for de Diagnosis and Treatment of Chronic Heart Failure of the European Society of Cardiology. Guidelines for the diagnosis and treatment of chronic heart failure. Eur Heart J. 2001;22:1527-60.

15. Swedberg K, Cleland J, Dargie H, Drexler H, Follath F, Komajda M, et al.. Guidelines for the diagnosis and treatment of chronic heart failure: executive summary (update 2005): The Task Force for the Diagnosis and Treatment of Chronic Heart Failure of the European Society of Cardiology. Eur Heart J. 2005;26:1115-40.

16. Hunt SA, Abraham WT, Chin MH, Feldman AM, Francis GS, Ganiats TG, et al.. ACC/ AHA 2005 Guideline Update for the diagnosis and management of chronic heart failure in the adult: a report of the American College of Cardiology/American Heart Association Task Force on Practice Guidelines. Circulation. 2005;112:e154-e235.

17. Grupo de Trabajo de Insuficiencia Cardíaca de la Sociedad Española de Medicina Interna (SEMI). La insuficiencia cardíaca en los servicios de medicina interna (estudio SEMI-IC). Med Clin (Barc). 2002;118: 605-10.

18. Cleland JGF, Swedberg K, Follath F, Komajda M, Cohen-Solal A Aguilar JC et al. The EuroHeart Failure Survey programme: a survey on the quality of care among patients with heart failure in Europe. Part 1: patients characteristics and diagnosis. Eur Heart J. 2003;24:442-63.

19. Komajda M, Lapuerta P, Hermans N, González-Juanatey JR, Van Veldhuisen DJ, Erdmann $\mathrm{E}$, et al.. Adherente to guidelines is a predictor of outcome in chronic heart failure: the MAHLER survey. Eur Heart J. 2005;26:1653-9.

20. Permanyer Miralda G, Soriano N, Brotons C, Moral I, Pinar J, Cascant P, et al.. Características basales y determinantes de la evolución en pacientes ingresados por insuficiencia cardiaca en un hospital general. Rev Esp Cardiol. 2002;55:571-8.

21. Galvao M, Kalman J, DeMarco T, Fonarow GC, Galvin C, Ghali JK, et al. Gender differences in in-hospital management and outcomes in patients with decompensated heart failure: analysis from the Acute Decompensated Heart Failure National Registry (ADHERE). J Card Fail. 2006;12:100-7.

22. Anguita Sánchez M, Investigadores del Registro BADAPIC. Características clínicas, tratamiento y morbimortalidad a corto plazo de pacientes con insuficiencia cardiaca controlados en consultas específicas de insuficiencia cardiaca. Resultados del Regiatro BADAPIC. Rev Esp Cardiol. 2004;57:1159-69.

23. Duaso E, Díez-Caballero M, Formiga F. Abordaje de la insuficiencia cardiaca en ancianos: una visión desde la atención primaria. Rev Esp Cardiol. 2006;59:290-1.

24. Barrios Alonso V, Peña Pérez G, González Juanatey JR, Alegría Ezquerra E, Lozano Vidal JV, Llisterri Caro JL, et al. Hipertensión arterial e insuficiencia cardiaca en las consultas de atención primaria y de cardiología en España. Rev Clin Esp. 2003;203:334-42.

25. Rodríguez Roca GC, Barrios Alonso V, Aznar Costa J, Llisterri Caro JL, Alonso Moreno FJ, Escobar Cervantes C et al., en representación de SEMERGEN, de la Sección de Cardiología Clínica y Extrahospitalaria de la Sociedad Española de Cardiología y de los investigadores del estudio CARDIOPRES. Características clínicas de los pacientes diagnosticados de insuficiencia cardiaca crónica asistidos en Atención Primaria. Estudio CARDIOPRES. Rev Clin Esp. 2007;207:337-40. 